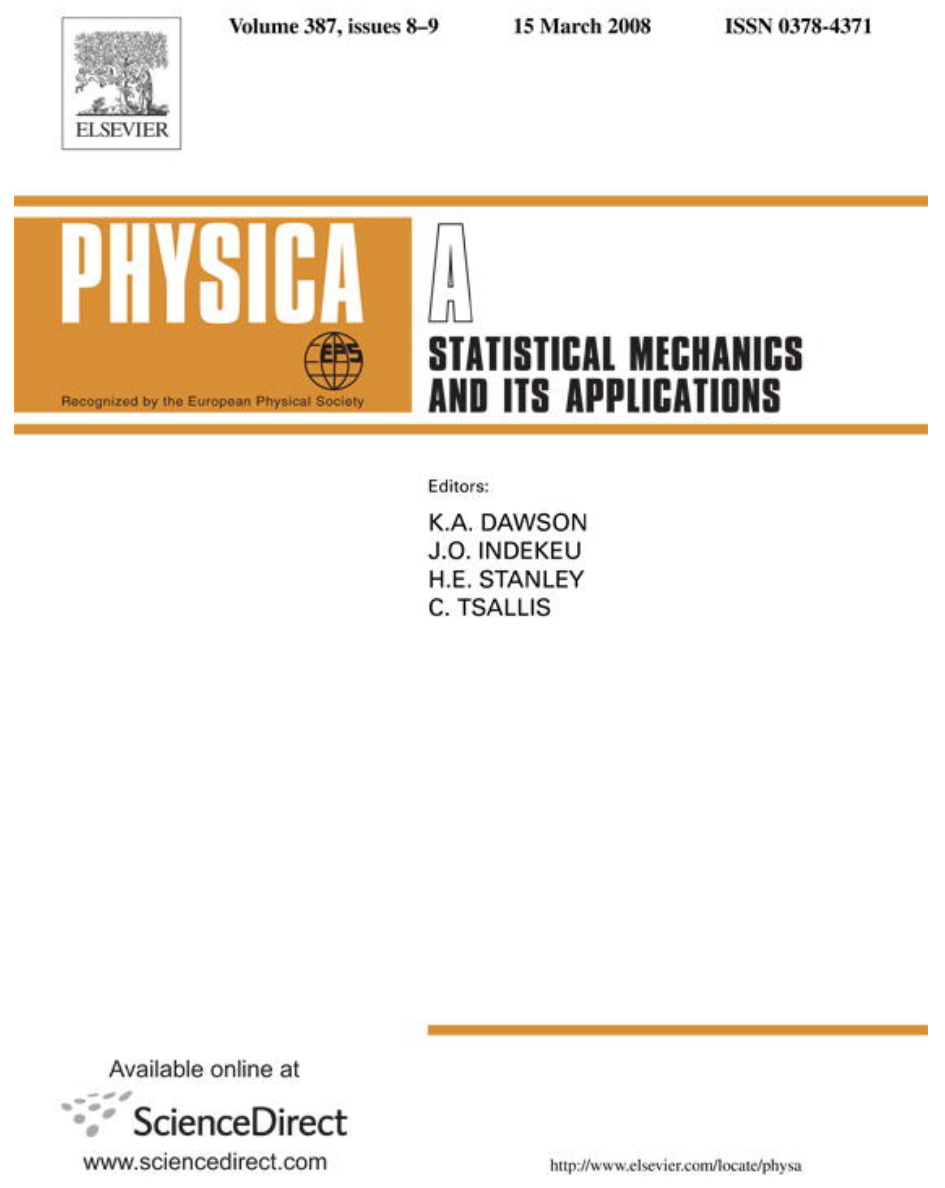

This article was published in an Elsevier journal. The attached copy

is furnished to the author for non-commercial research and education use, including for instruction at the author's institution, sharing with colleagues and providing to institution administration.

Other uses, including reproduction and distribution, or selling or licensing copies, or posting to personal, institutional or third party websites are prohibited.

In most cases authors are permitted to post their version of the article (e.g. in Word or Tex form) to their personal website or institutional repository. Authors requiring further information regarding Elsevier's archiving and manuscript policies are encouraged to visit:

http://www.elsevier.com/copyright 


\title{
Multifractal spectrum of phase space related to generalized thermostatistics
}

\author{
A.I. Olemskoi ${ }^{\mathrm{a}, *}$, V.O. Kharchenko ${ }^{\mathrm{b}}$, V.N. Borisyuk ${ }^{\mathrm{c}}$ \\ ${ }^{a}$ Institute of Applied Physics, Nat. Acad. Sci. of Ukraine, 58, Petropavlovskaya St., 40030 Sumy, Ukraine \\ ${ }^{\mathrm{b}}$ Institute of Magnetism, Nat. Acad. Sci. of Ukraine, 36-B, Vernadsky St., 03680 Kyiv, Ukraine \\ ${ }^{\mathrm{c}}$ Sumy State University, 2, Rimskii-Korsakov St., 40007 Sumy, Ukraine
}

Received 3 September 2007; received in revised form 3 November 2007

Available online 3 December 2007

\begin{abstract}
We consider a self-similar phase space with specific fractal dimension $d$ being distributed with spectrum function $f(d)$. Related thermostatistics is shown to be governed by the Tsallis formalism of the non-extensive statistics, where the non-additivity parameter equals to $\bar{\tau}(q) \equiv 1 / \tau(q)>1$, and the multifractal function $\tau(q)=q d_{q}-f\left(d_{q}\right)$ is the specific heat determined with multifractal parameter $q \in[1, \infty]$. At that, the equipartition law is shown to take place. Optimization of the multifractal spectrum function $f(d)$ arrives at the relation between the statistical weight and the system complexity. It is shown that the statistical weight exponent $\tau(q)$ can be modeled by hyperbolic tangent deformed in accordance with both Tsallis and Kaniadakis exponential functions to describe arbitrary multifractal phase space explicitly. The spectrum function $f(d)$ is proved to increase monotonically from minimum value $f=-1$ at $d=0$ to maximum one $f=1$ at $d=1$. At the same time, the number of monofractals increases with the growth of the phase-space volume at small dimensions $d$ and falls down in the limit $d \rightarrow 1$.
\end{abstract}

(C) 2007 Elsevier B.V. All rights reserved.

PACS: 05.20.Gg; 05.45.Df; 05.70.Ce

Keywords: Phase space; Multifractal spectrum; Statistical weight

\section{Introduction}

A generalization of the statistical mechanics onto the non-extensive thermostatistics is known to be based on the deformation procedure of both logarithmic and exponential functions [1-3]. The simplest way to introduce these functions into the thermostatistics scheme is to consider the equation of motion for dimensionless volume $\gamma=\Gamma /(2 \pi \hbar)^{6 N}$ of the supported phase space ( $\hbar$ and $N$ being the Dirac-Planck constant and particle number, respectively). In the course of evolution of the ensemble with statistical weight $w=w(\gamma)$ and entropy $S=S(t)$, the variation rate of the phase-space volume $\gamma=\gamma(t)$ is supposed [3] to be governed by the equation

$$
\frac{\mathrm{d} \gamma}{\mathrm{d} t}=w(\gamma) \frac{\mathrm{d} S}{\mathrm{~d} t} .
$$

\footnotetext{
* Corresponding author.

E-mail addresses: alex@ufn.ru (A.I. Olemskoi), vasiliy@imag.kiev.ua (V.O. Kharchenko), vadimborisyuk@ yahoo.com (V.N. Borisyuk).
} 
Following from here relation $\mathrm{d} S=\mathrm{d} \gamma / w(\gamma)$ gives the entropy corresponding to the whole statistical weight $W$ :

$$
S(W)=\int_{\gamma(1)}^{\gamma(W)} \frac{\mathrm{d} \gamma}{w(\gamma)}
$$

Here, we take into account that entropy of a single state $W=1$ vanishes, i.e., $S(1)=0$.

In the case of the smooth phase space, one has trivial relation $w(\gamma)=\gamma$ whose insertion into Eq. (2) arrives at the Boltzmann entropy $S=\ln W$. However, complex systems have fractal phase space with the dimension $D<6 N$, so that the relation between the statistical weight and the corresponding volume should be generalized by the power-law dependence

$$
w(\gamma)=\gamma^{d}
$$

where the specific fractal dimension $d \equiv D / 6 N \leq 1$ is introduced as the exponent. Insertion of Eq. (3) into the integral (2) gives the expression ${ }^{1}$

$$
S(W)=\bar{d} \ln _{2-\bar{d}}(W), \quad \ln _{q}(x) \equiv \frac{x^{1-q}-1}{1-q}
$$

which is reduced to the Tsallis $\operatorname{logarithm} \ln _{q}(x)$ where the non-additivity parameter $q$ is replaced by the difference $2-\bar{d}$ with $\bar{d} \equiv 1 / d \geq 1$ being the inverse value of the specific fractal dimension $d$ of the phase space. Naturally, this expression gives the Boltzmann entropy in the limit $d \rightarrow 1$.

Above formalism is based on the proposition that the phase space relates to a monofractal set determined by single dimension $d$. However, the considerations [4-6] show that a complex system behaviour can be determined by the phase-space geometry, being much more complicated, in particular multifractal. In this connection, we aim to generalize the Tsallis thermostatistics onto the multifractal phase space with a spectrum $f(d)$. Such a generalization for arbitrary distribution $f(d)$ is carried out in Section 2. Related discussion shows that physical representation of the thermostatistics based on the multifractal phase space demands of the passage from input distribution to escort one. An optimization procedure of the spectrum $f(d)$ is considered in Section 3 to derive the relation between the statistical weight and the system complexity. In Section 4 we show that the monotonically increasing mass exponent $\tau(q)$, being free energy of the multifractal set [7], is presented by the hyperbolic tangent deformed in accordance with both Tsallis and Kaniadakis procedures, which allow to describe explicitly arbitrary multifractal phase space. Section 5 is devoted to consideration of the multifractal spectrum $f(d)$ which determines the number of monofractals within the multifractal with the specific dimension $d$. Section 6 concludes our consideration.

\section{Thermostatistics related to a multifractal phase space}

According to the self-similarity condition the specific statistical weight of the system under consideration is given by the power-law function [8]

$$
\varpi_{q}(\gamma)=\gamma^{q d}
$$

where $q$ is the multifractal exponent, $d \equiv D / 6 N \leq 1$ is the specific fractal dimension. This function should be multiplied by the number of monofractals with dimension $d$

$$
\mathcal{N}_{d}(\gamma)=\gamma^{-f(d)}
$$

which are contained within the multifractal whose spectrum is determined by a function $f(d)$. As a result, the whole statistical weight, being the multifractal measure, takes the form

$$
w_{q}(\gamma) \equiv \int_{0}^{1} \varpi_{q}(\gamma) \mathcal{N}_{d}(\gamma) \rho(d) \mathrm{d} d=\int_{0}^{1} \gamma^{q d-f(d)} \rho(d) \mathrm{d} d
$$

where $\rho(d)$ is a density distribution over dimensions $d$. Using the method of the steepest descent, we arrive at the power law

\footnotetext{
1 This expression is equivalent to Eq. (16) in Ref. [3] since W in our manuscript denotes $\mathcal{N}$ given by (11) in Ref. [3].
} 


$$
w_{q}(\gamma) \simeq \gamma^{\tau(q)}
$$

which generalizes the simplest relation (3) due to the replacement of the bare fractal dimension $d$ by the multifractal function

$$
\tau(q)=q d_{q}-f\left(d_{q}\right),
$$

being the mass exponent [8]. Here, the specific fractal dimension $d_{q}$ relates to the given parameter $q$ to be defined by the following conditions of the steepest-descent method:

$$
\left.\frac{\mathrm{d} f}{\mathrm{~d} d}\right|_{d=d_{q}}=q,\left.\quad \frac{\mathrm{d}^{2} f}{\mathrm{~d} d^{2}}\right|_{d=d_{q}}<0 .
$$

The above consideration shows that the passage from the monofractal phase space to the multifractal one is obtained by replacement of the single dimension $d$ by the monotonically increasing function $\tau(q)$, such as $\tau(0)=-1$ and $\tau(1)=0$. The limit behaviour of the function $\tau(q)$ is characterized by the asymptotics [8]

$$
\tau \propto(q-1) \text { at } 0 \leq q-1 \ll 1, \quad \tau \simeq 1 \text { at } q \rightarrow \infty .
$$

A physical domain of the $q$ parameter variation is bounded by the condition $q \geq 1$ which ensures positive values of the function $0 \leq \tau(q) \leq 1$ to guarantee growth of the specific statistical weight (8) with increasing phase-space volume.

According to the entropy expression (4) we can use the well-known Tsallis formalism of the non-extensive statistical mechanics where the difference $2-\bar{\tau}(q)$ with $\bar{\tau}(q) \equiv 1 / \tau(q)>1$ plays a role of the non-additivity parameter. Thus, the entropy in dependence of the probability distribution $P_{i}$ has the form [1]

$$
S_{q}=-\sum_{i=1}^{W_{q}} P_{i} \frac{P_{i}^{\bar{\tau}-1}-1}{\bar{\tau}-1}
$$

where the statistical weight $W_{q}$ is related to a given value $q$ of the multifractal exponent. By accounting the normalization conditions and the definition of the internal energy $E_{q}$

$$
\sum_{i=1}^{W_{q}} P_{i}=1, \quad E_{q}=\sum_{i=1}^{W_{q}} \varepsilon_{i} P_{i}^{\bar{\tau}(q)},
$$

the expression (12) arrives at the generalized distribution over energy levels $\varepsilon_{i}$ as follows:

$$
P_{i}=Z_{q}^{-1} \exp _{\bar{\tau}(q)}\left(-\beta \varepsilon_{i}\right), \quad Z_{q} \equiv \sum_{i=1}^{W_{q}} \exp _{\bar{\tau}(q)}\left(-\beta \varepsilon_{i}\right) .
$$

Here, $Z_{q}$ is the partition function, $\beta$ is the Lagrange multiplier, not being the physical temperature, and the deformed exponential function is determined by the expression

$$
\exp _{\bar{\tau}}(x) \equiv \begin{cases}{[1+(\bar{\tau}-1) x]^{\frac{1}{\bar{\tau}-1}}} & \text { at } 1+(\bar{\tau}-1) x>0, \\ 0 & \text { otherwise. }\end{cases}
$$

Parameter $q$ characterizes here the multifractal spectrum through the function $\tau(q)$ and should not be confused with the non-additivity parameter of the Tsallis thermostatistics, which is denoted here by $\bar{\tau}(q) \equiv 1 / \tau(q)$.

Thermodynamic functions of the model under consideration can be found according to the Tsallis non-extensive scheme [1]. However, related expressions are very cumbersome even in the simplest case of the ideal gas [9-11] and take the usual form only within the slightly non-extensive limit [12]. At the same time, developed scheme allows to use the thermodynamic formalism of multifractal objects [13], within the which the multifractal exponent $q$ plays the role of a state parameter. If the dependence $\tau(q)$ has some singularities, then variation in $q$ may arrive at phase transitions. It is worthwhile to stress that the developed scheme arrives directly at the related singularities of thermodynamic 
function types of the internal energy (see below Eq. (23), the entropy (cf. Eq. (4))

$$
S_{q}=\bar{\tau}(q) \ln _{2-\bar{\tau}(q)}\left(W_{q}\right), \quad \bar{\tau}(q) \equiv 1 / \tau(q)
$$

and the free energy

$$
F_{q}=E_{q}-T S_{q} .
$$

According to Ref. [9], the physical distribution is not the input probability (14), but the escort one

$$
\mathcal{P}\left(\varepsilon_{i}\right) \equiv \frac{P^{\bar{\tau}(q)}\left(\varepsilon_{i}\right)}{\sum_{i=1}^{W_{q}} P^{\bar{\tau}(q)}\left(\varepsilon_{i}\right)} .
$$

It corresponds to the condition

$$
\sum_{i=1}^{W_{q}}\left(\varepsilon_{i}-E_{q}\right) P^{\bar{\tau}(q)}\left(\varepsilon_{i}\right)=0
$$

instead of the second Eq. (13). The difference of the distribution (14), the related probability

$$
\mathcal{P}\left(\varepsilon_{i}\right)=\mathcal{Z}_{q}^{-1} \exp _{\tau(q)}\left[-\bar{\tau}(q)\left(\varepsilon_{i}-E_{q}\right) / T\right]
$$

is determined with the physical temperature $T$.

In the case of continuous energy spectrum characterized with the density distribution $\rho(\varepsilon)$, the internal energy related to the condition (19) takes the form

$$
E_{q}=\int_{-\infty}^{\infty} \varepsilon \mathcal{P}(\varepsilon) \rho(\varepsilon) \mathrm{d} \varepsilon .
$$

Extreme value of $E_{q}$ is reached at the condition

$$
\frac{\rho^{\prime}(\varepsilon)}{\rho(\varepsilon)} \simeq-\frac{\mathcal{P}^{\prime}(\varepsilon)}{\mathcal{P}(\varepsilon)}
$$

where prime denotes differentiation over $\varepsilon$. Usually, the density function is reduced to the power law $\rho(\varepsilon) \sim \varepsilon^{c N}$, $c \sim 1$, so that $\rho^{\prime}(\varepsilon) / \rho(\varepsilon) \simeq c N / \varepsilon$. Then, by using the distribution (20), the condition (22) taken at $\varepsilon=E_{q}$ arrives at the equipartition law

$$
E_{q}=c \tau(q) N T
$$

where the value $c \tau(q)$ is the specific heat.

\section{Optimization of the multifractal spectrum}

Up to now, we supposed that the multifractal spectrum $f(d)$ is arbitrary. If it is optimized at the normalization condition

$$
\int_{0}^{1} f(d) \mathrm{d} d=1,
$$

one has to minimize the expression

$$
\tilde{S}_{q}\{f(d)\}=\int_{\gamma(1)}^{\gamma\left(W_{q}\right)}\left[\int_{0}^{1} \gamma^{q d-f(d)} \rho(d) \mathrm{d} d\right]^{-1} \mathrm{~d} \gamma-\frac{\Sigma^{2}}{2}\left[\int_{0}^{1} f(d) \mathrm{d} d-1\right]
$$



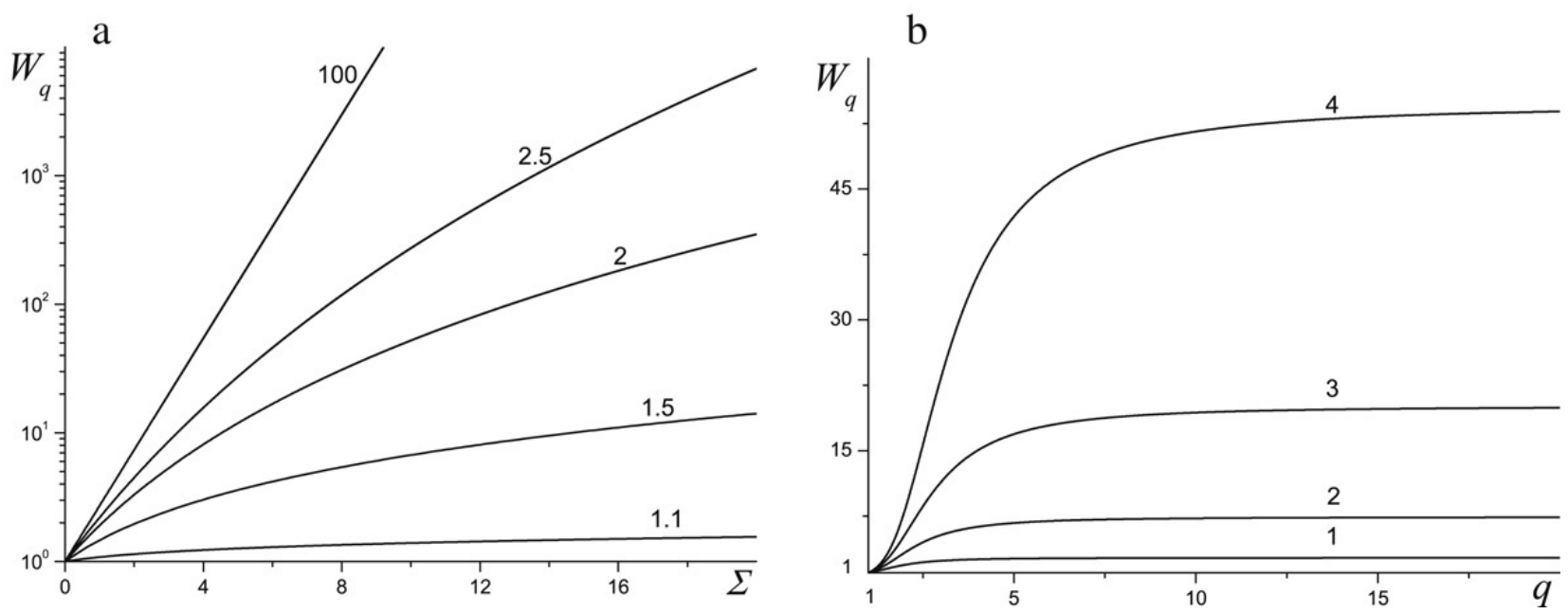

Fig. 1. Dependencies of the statistical weight on the complexity (a) and multifractal exponent (b) at the exponent (31) (numbers near curves on the left panel show values $q$, on the right one-values $\Sigma$ ).

where we take into account Eqs. (2) and (7), and $\Sigma$ determines the Lagrange multiplier. As a result, we arrive at the equality

$$
\int_{\gamma(1)}^{\gamma\left(W_{q}\right)} \gamma^{\{[q d-f(d)]-2 \tau(q)\}} \ln \gamma \mathrm{d} \gamma=\frac{\Sigma^{2}}{2}
$$

whose integration gives, taking into account Eq. (8), the transcendental equation

$$
\begin{aligned}
& \frac{1}{2}\left[\Sigma \tau(q) \mathcal{T}_{q}(d)\right]^{2}-W_{q}^{\mathcal{T}_{q}(d)}\left[\mathcal{T}_{q}(d) \ln \left(W_{q}\right)-1\right]-1=0, \\
& \mathcal{T}_{q}(d) \equiv \bar{\tau}(q)\{1-2 \tau(q)+[q d-f(d)]\} .
\end{aligned}
$$

This equation is written in the form, which can be used as a given function either the spectrum function $f(d)$ or the exponent dependence $\tau(q)$. In the latter case, we find initially the dependence $q(d)$ from the equation

$$
\left.\frac{\mathrm{d} \tau}{\mathrm{d} q}\right|_{q=q(d)}=d
$$

being conjugated to Eq. (10). Then, inserting this dependence into Eq. (28), we arrive at the trivial expression

$$
\mathcal{T}(q) \equiv \mathcal{T}_{q}(d(q))=\bar{\tau}(q)-1
$$

which when used in Eq. (27) allows to determine the dependence of the statistical weight $W_{q}$ versus the complexity $\Sigma$ at a given function $\tau(q)$. A typical form of this dependence at the mass exponent

$$
\tau=\frac{q-1}{\sqrt{1+(q-1)^{2}}}
$$

is shown in Fig. 1. It is seen that the statistical weight increases monotonically as complexity grows, similarly with increasing multifractal exponent.

In the limit of smooth phase space, when $q \rightarrow \infty, d \rightarrow 1, \mathcal{T}(q) \rightarrow 0$, one obtains the usual expression for the statistical weight of the complex system

$$
W_{\infty}=\mathrm{e}^{\Sigma_{\infty}}, \quad \Sigma_{\infty} \equiv \sigma_{\infty} N
$$

which is determined by the specific complexity $\sigma_{\infty}$ per one particle. At small deviation off the minimum complexity $\left(\Sigma-\Sigma_{\infty} \ll \Sigma_{\infty}\right)$ and light multifractality $\left(1-\tau(q) \ll \Sigma_{\infty}^{-1}\right)$, linearized Eq. (27) gives 


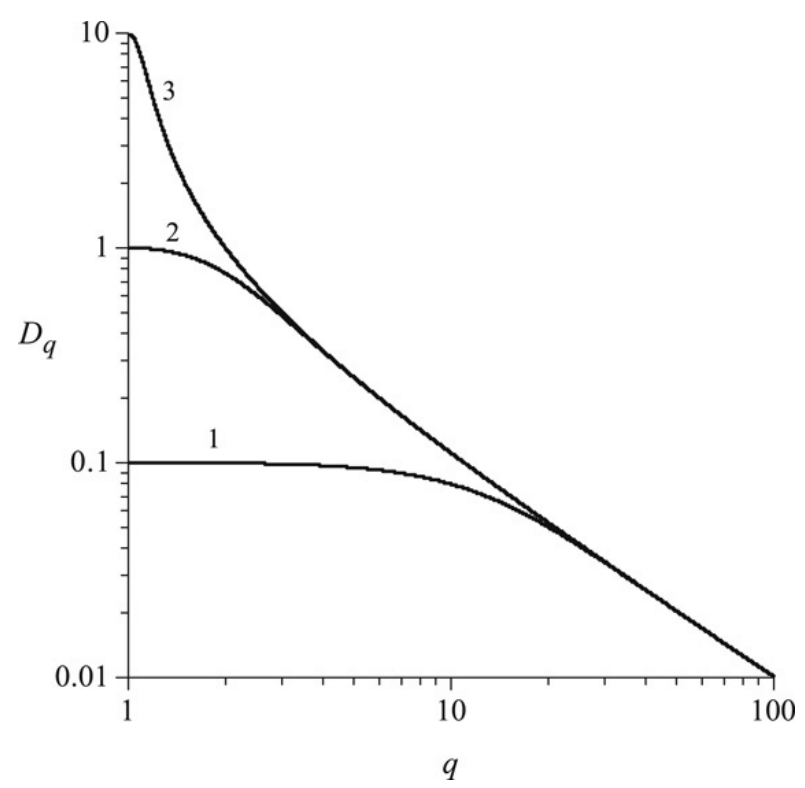

Fig. 2. Multifractal dimension function related to the dependence (35) (curves $1,2,3$ correspond to $\mathcal{D}=0.1,1.0,10$ ).

$$
\begin{aligned}
& W_{q} \simeq W_{\tau(q)}\left\{1+\tau(q)\left[1-\frac{2}{3}(1-\tau(q)) \Sigma_{\infty}\right]\left(\Sigma-\Sigma_{\infty}\right)\right\}, \\
& W_{\tau(q)} \equiv \exp \left\{\tau(q) \Sigma_{\infty}\left[1-\frac{1}{3}(1-\tau(q)) \Sigma_{\infty}\right]\right\}, \quad 1 \ll q<\infty .
\end{aligned}
$$

In the opposite case $\tau(q) \ll 1$, one has, within the logarithmic accuracy,

$$
W_{q} \simeq\left[\alpha \tau(q) \Sigma^{2}\right]^{\tau(q)}, \quad \alpha \sim 1, q-1 \ll 1 .
$$

As shown in the above findings, optimization of the multifractal spectrum, obeying the normalization condition (24), gives the dependence of the statistical weight $W_{q}$ versus the system complexity $\Sigma$ at a given multifractal exponent $\tau(q)$. Naturally, relations (27) and (30) enable one to solve the inverse problem-to find the dependence $\tau(q)$ at given function $W_{q}(\Sigma)$. However, definition of the dependence $W_{q}(\Sigma)$ leads to very complicated problem. It is more convenient to use a modeling function $\tau(q)$ bounded with asymptotics (11) and then to find the statistical weight $W_{q}$. Within this algorithm, in the following section we model the multifractal spectrum on the basis of the procedure of both the Tsallis and Kaniadakis deformations. It appears that such deformations give the whole set of functions $f(d)$ to present all possible types of multifractal spectra.

\section{Analytical modeling multifractal spectrum}

As the simplest case, we can take the function $\tau(q)$ in the form

$$
\tau=\tanh [\mathcal{D}(q-1)]
$$

being determined by parameter $\mathcal{D}>0$ and argument $q \in[1, \infty]$. According to Fig. 2 related multifractal dimension function [8]

$$
D_{q}=\frac{\tau(q)}{q-1}
$$

monotonically decreases from the maximum value $D_{0}=\mathcal{D}$ to the minimum one $D_{\infty}=0$ with increase in $q$. However, the maximum value of the fractal dimension $D_{q}$ is fixed by the magnitude $D_{0}=1$, so that one should put $\mathcal{D}=1$ in the dependence (35). As a result, it takes quite trivial form.

Due to the $\tau(q)$ function increasing monotonically within the narrow interval $[0,1]$, one has a scanty choice of its analytical models. To set a possible representation of $\tau(q)$ one can use a deformation of the hyperbolic tangent (35) at 


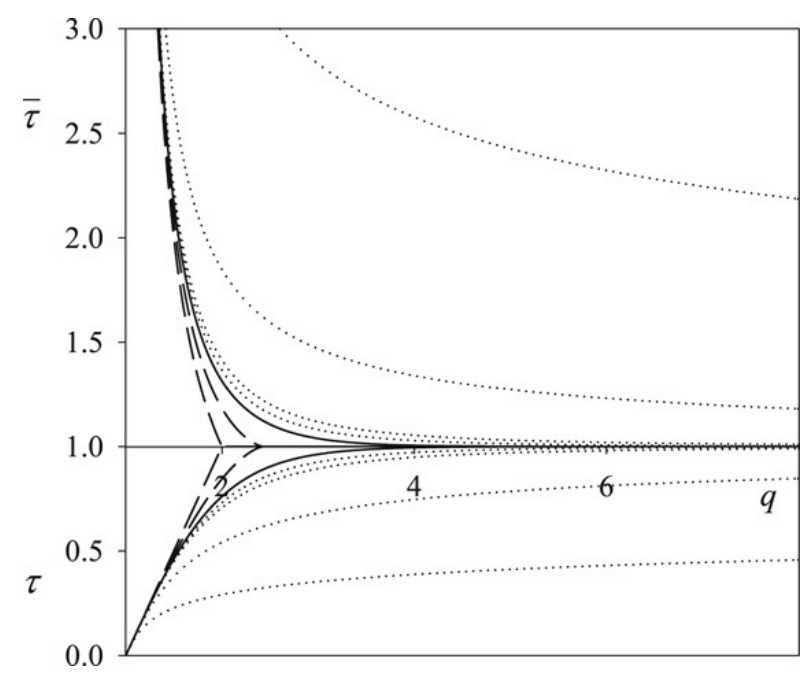

Fig. 3. The $q$-dependencies of the multifractal exponent $\tau$ and its inverse value $\bar{\tau}=1 / \tau$ (solid line corresponds to $\kappa=0$, dashed curves relate to the Tsallis deformation with $\kappa=0.7,1$; dotted lines correspond to the Kaniadakis one at $\kappa=0.7,1,3,10$ ).

$\mathcal{D}=1$. By now, two analytical procedures of such deformation are extensively popularized. The first of them is based on the Tsallis exponential form [1]

$$
\exp _{\kappa}(x) \equiv \begin{cases}(1+\kappa x)^{1 / \kappa} & \text { at } 1+\kappa x>0 \\ 0 & \text { otherwise }\end{cases}
$$

where deformation parameter $\kappa$ takes positive values. The second procedure has been proposed by Kaniadakis [14] to determine the deformed exponential form

$$
\exp _{\kappa}(x) \equiv\left(\kappa x+\sqrt{1+\kappa^{2} x^{2}}\right)^{1 / \kappa}
$$

By using these definitions, the deformed tangent (35) takes the form

$$
\tau_{\kappa}(q)=\tanh _{\kappa}(q-1) \equiv \frac{\exp _{\kappa}(q-1)-\exp _{\kappa}(1-q)}{\exp _{\kappa}(q-1)+\exp _{\kappa}(1-q)}
$$

where the multifractal exponent $q$ varies within the domain $[1, \infty]$.

The $q$-dependencies of the multifractal exponent $\tau(q)$ and its inverse value $\bar{\tau}(q)=1 / \tau(q)$ are shown in Fig. 3 at different magnitudes of both the Tsallis and Kaniadakis deformation parameters $\kappa$. (The $\kappa$ values are picked out in such a manner to cover uniformly the panels of Figs. 3-5 with related curves.) It is principally important, the first of these deformations arrive at more fast variations of both exponents $\tau(q)$ and $\bar{\tau}(q)$ in comparison with the nondeformed hyperbolic tangent $\tau_{0}=\tanh _{0}(q-1)$, whereas the Kaniadakis deformation slows down these variations with increase in $\kappa$.

A characteristic peculiarity of the Tsallis deformation consists in breaking dependencies $\tau(q), \bar{\tau}(q)$ in the point $q_{0}=(1+\kappa) / \kappa$ where the second terms in both numerator and denominator of the definition (39) take the zero value. As a result, the multifractal exponent (9) takes the asymptotics

$$
\tau_{\kappa}^{(T s)} \simeq \begin{cases}(q-1)-\frac{1-\kappa^{2}}{3}(q-1)^{3} & \text { at } 0<q-1 \ll 1, \\ 1-2(\kappa / 2)^{1 / \kappa}\left(q_{0}-q\right)^{1 / \kappa} & \text { at } 0<q_{0}-q \ll q_{0} .\end{cases}
$$

For $\kappa=1$ the dependence $\tau_{1}^{(T s)}(q)$ takes the simplest form: $\tau_{1}^{(T s)}=q-1$ at $1 \leq q \leq 2$, and $\tau_{1}^{(T s)}=1$ at $q>2$. It is worth noting that the Tsallis deformation parameter cannot take values $\kappa>1$ because they are related to the fractal dimensions $D_{q}>1$ at $q \neq 0$. 

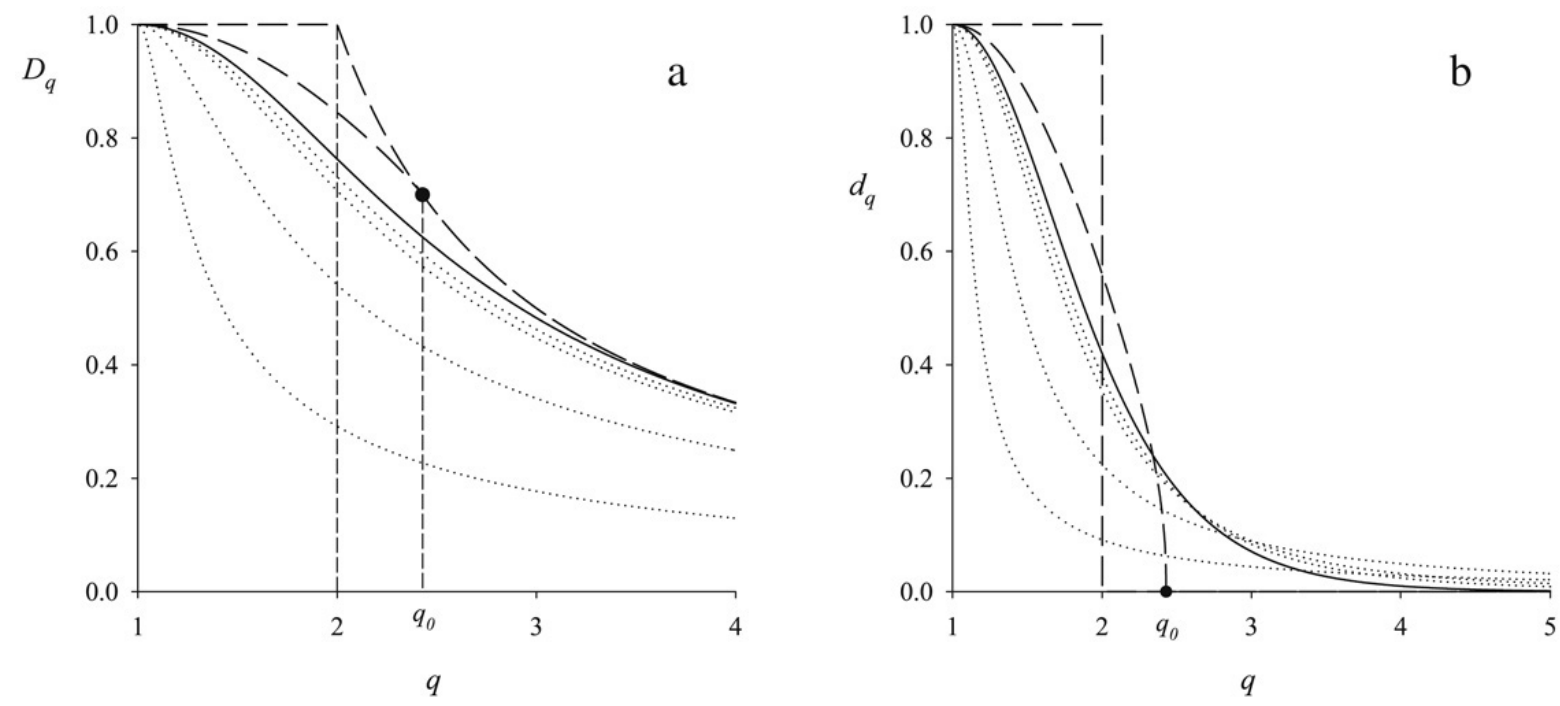

Fig. 4. Spectra of fractal (a) and specific (b) dimensions of the phase space (solid line corresponds to $\kappa=0$, dashed curves relate to the Tsallis deformation with $\kappa=0.7,1$; dotted lines correspond to the Kaniadakis one at $\kappa=0.7,1,3,10$ ).

In the case of the Kaniadakis deformation, the multifractal exponent $\tau_{\kappa}(q)$ varies smoothly to be characterized by the following asymptotics:

$$
\tau_{\kappa}^{(K)} \simeq \begin{cases}(q-1)-\frac{2+\kappa^{2}}{6}(q-1)^{3} & \text { at } 0<q-1 \ll 1, \\ 1-2[2 \kappa(q-1)]^{-2 / \kappa} & \text { at } \kappa(q-1) \gg 1 .\end{cases}
$$

In contrast to the Tsallis case, here the deformation parameter can take arbitrary values to give the simplest dependence (31) at $\kappa=1$.

The fractal dimension (36) as a function of the $q$ exponent falls down monotonically as shown in Fig. 4(a). According to Eqs. (40), in the case of the Tsallis deformation, one has a broken dependence $D(q)$, being characterized by the asymptotics

$$
D_{q}^{(T s)} \simeq \begin{cases}1-\frac{1-\kappa^{2}}{3}(q-1)^{2} & \text { at } 0<q-1 \ll 1, \\ \frac{1}{q-1}-2(\kappa / 2)^{1 / \kappa} \frac{\left(q_{0}-q\right)^{1 / \kappa}}{q-1} & \text { at } 0<q_{0}-q \ll q_{0} .\end{cases}
$$

In the limit case $\kappa=1$, the phase space is smooth $\left(D_{q}^{(T s)}=1\right)$ within the interval $1 \leq q \leq 2$. For the Kaniadakis deformation, the fractal dimension $D_{q}^{(K)}$ is given by smoothly falling down curve whose slope increases with the deformation parameter growth. According to Eqs. (41), in this case, one has the asymptotics

$$
D_{q}^{(K)} \simeq \begin{cases}1-\frac{2+\kappa^{2}}{6}(q-1)^{2} & \text { at } 0<q-1 \ll 1, \\ (q-1)^{-1}-2(2 \kappa)^{-2 / \kappa}(q-1)^{-(\kappa+2) / \kappa} & \text { at } \kappa(q-1) \gg 1 .\end{cases}
$$

At $\kappa=1$, the typical dependence related to Eq. (31) is of the form

$$
D_{q}^{(K)}=\left[1+(q-1)^{2}\right]^{-1 / 2} .
$$

\section{Multifractal spectrum}

At the given multifractal exponent $\tau(q)$, the spectrum function $f(d)$ is defined by the Legendre transformation (9) where the specific dimension reads 


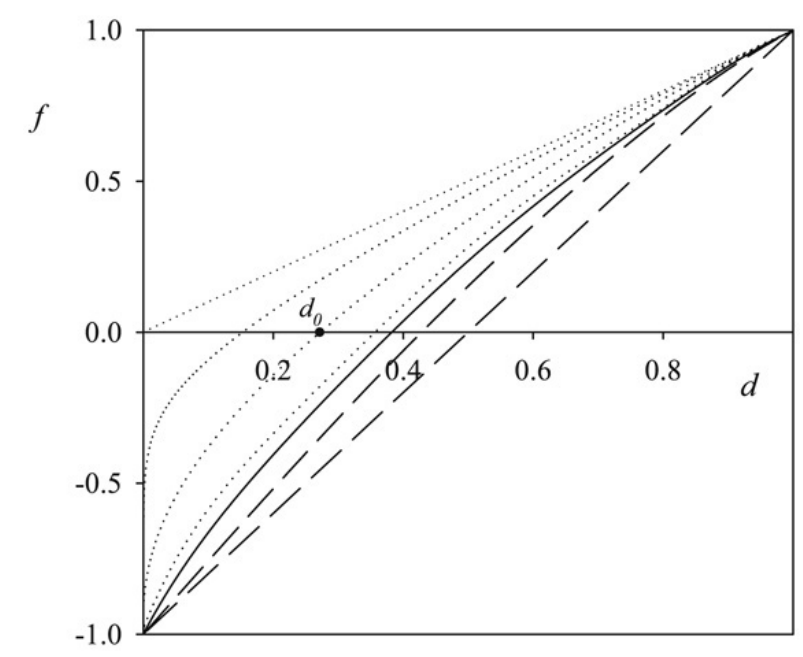

Fig. 5. Spectrum function of the multifractal phase space (solid line corresponds to $\kappa=0$, dashed curves relate to the Tsallis deformation with $\kappa=0.7,1$; dotted lines correspond to the Kaniadakis one at $\kappa=1,3,10, \infty)$.

$$
d_{q}=\frac{\mathrm{d} \tau}{\mathrm{d} q}
$$

As shown in Fig. 4(b), the dependence $d_{q}$ has monotonically falling down form to take the value $d_{q}=0$ at $q>q_{0} \equiv(1+\kappa) / \kappa$ for the Tsallis deformation. In this case, asymptotic behaviour is characterized by Eq. (40), according to which one obtains

$$
d_{q}^{(T s)} \simeq \begin{cases}1-\left(1-\kappa^{2}\right)(q-1)^{2} & \text { at } 0<q-1 \ll 1, \\ (\kappa / 2)^{(1-\kappa) / \kappa}\left(q_{0}-q\right)^{(1-\kappa) / \kappa} & \text { at } 0<q_{0}-q \ll q_{0} .\end{cases}
$$

In the limit $\kappa \rightarrow 1$, the dependence $d^{(T s)}(q)$ takes the step-like form being $d_{q}=1$ within the interval $1 \leq q \leq 2$ and $d_{q}=0$ otherwise.

For the Kaniadakis deformation, Eqs. (41) arrive at the asymptotics

$$
d_{q}^{(K)} \simeq \begin{cases}1-\frac{2+\kappa^{2}}{2}(q-1)^{2} & \text { at } 0<q-1 \ll 1, \\ 2^{2(\kappa-1) / \kappa}[\kappa(q-1)]^{-(2+\kappa) / \kappa} & \text { at } \kappa(q-1) \gg 1 .\end{cases}
$$

The typical behaviour is presented by the dependence

$$
d_{q}^{(K)}=\left[1+(q-1)^{2}\right]^{-3 / 2}
$$

related to $\kappa=1$.

The multifractal spectrum is defined by the equality

$$
f(d)=d q_{d}-\tau\left(q_{d}\right)
$$

being conjugated to Eq. (9). Here, the specific multifractal exponent $q_{d}$ is determined by Eq. (45) which arrives at the limit relations (46) and (47). With their using, one obtains the asymptotics

$$
f^{(T s)} \simeq \begin{cases}d-\frac{2}{3}\left(1-\kappa^{2}\right)^{-1 / 2}(1-d)^{3 / 2} & \text { at } 0<1-d \ll 1, \\ -\left[1+2(1 / \kappa-1) d^{1 /(1-\kappa)}\right]+(1+1 / \kappa) d & \text { at } d \ll 1\end{cases}
$$




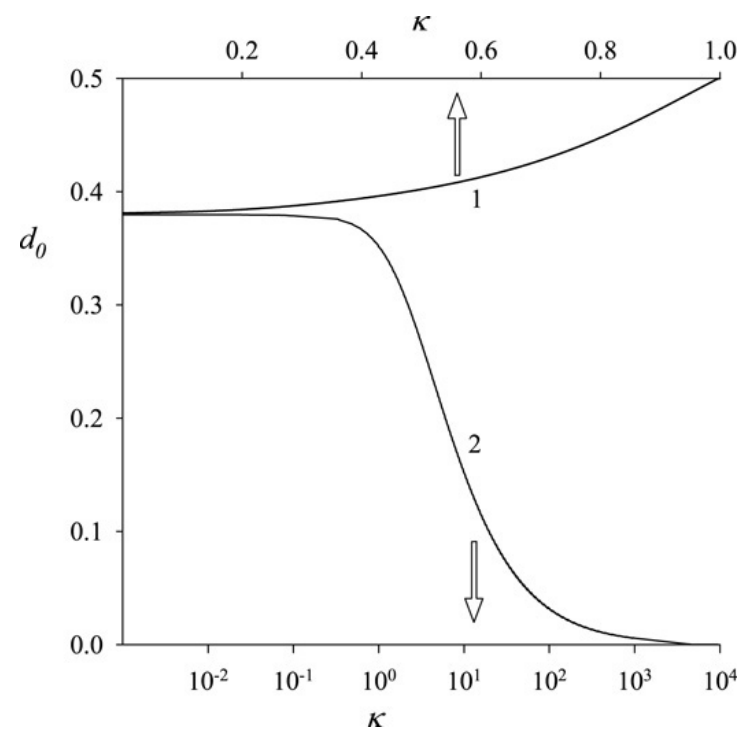

Fig. 6. Dimension $d_{0}$ related to the condition $f(d)=0(\mathcal{N}=1)$ as function of the parameter $\kappa$ (curve 1 corresponds to the Tsallis deformation, curve 2 corresponds to the Kaniadakis one; positive values $f(d)$ relate to the domain $d>d_{0}$ ).

for the Tsallis deformation, and the relations

$$
f^{(K)} \simeq \begin{cases}d-\frac{2}{3}\left(1+\frac{\kappa^{2}}{2}\right)^{-1 / 2}(1-d)^{3 / 2} & \text { at } 0<1-d \ll 1, \\ -\left[1-2^{(\kappa-4) /(2+\kappa)}(1+2 / \kappa) d^{2 /(2+\kappa)}\right]+d & \text { at } d \ll 1,\end{cases}
$$

characterizing the Kaniadakis deformation.

As shown in Fig. 5, for finite deformation parameters $\kappa<\infty$, a spectrum function increases monotonically, taking the minimum value $f=-1$ at $d=0$ and the maximum one $f=1$ at $d=1$. Besides, the derivative $f^{\prime} \equiv \mathrm{d} f / \mathrm{d} d$ equals to $f^{\prime}(0)=\infty$ on the left boundary and $f^{\prime}(1)=1$ on the right one. It is significant, that the whole set of the spectrum functions is bounded by the limit dependencies $f^{(T s)}=2 d-1$ and $f^{(K)}=d$, the first of which relates to limit magnitude of the Tsallis deformation parameter $\kappa=1$, whereas the second one corresponds to the Kaniadakis limit $\kappa=\infty$. A typical form of the spectrum function is presented by the dependencies

$$
f^{(K)}= \begin{cases}-d \ln \left(\frac{\sqrt{d}}{1+\sqrt{1-d}}\right)+(d-\sqrt{1-d}) & \text { at } \kappa=0, \\ -\left(1-d^{2 / 3}\right)^{3 / 2}+d & \text { at } \kappa=1 .\end{cases}
$$

It may seem, at the first glance, that negative values of the spectrum function $f(d)$ has no physical meaning. To clear up this problem, let us take the set of monofractals with the specific dimension $d=0$. Obviously, such monofractals relate to the whole set of the phase-space points, whose number equals to the dimensionless volume $\gamma$. Just such result gives the definition (2) in the point $d=0$ where $f=-1$. On the other hand, in the opposite case $d=1$, where $f=1$, the number of monofractals with volume $\gamma$ is equal to $\mathcal{N}_{1}=\gamma^{-1}$ which gives one multifractal of the same volume $\gamma$. At the same time, a single monofractal is contained in the multifractal at condition $f(d)=0$, which takes place at the specific dimension $d_{0}$, whose dependence on the deformation parameter $\kappa$ is shown in Fig. 6.

The dependence of the number $\mathcal{N}$ of monofractals containing in the phase-space volume $\gamma$ related to the multifractal with the specific dimension $d$ is shown in Fig. 7. It is seen, the number $\mathcal{N}$ increases with the $\gamma$ volume growth at small dimensions $d$, whereas in the limit $d \rightarrow 1$ the dependence $\mathcal{N}(\gamma)$ decreases to give infinitely increasing numbers $\mathcal{N}$ at $\gamma \rightarrow 0$. This increase grows monotonically with both a decrease in the Tsallis deformation parameter $\kappa$ and an increase in the Kaniadakis deformation parameter. 


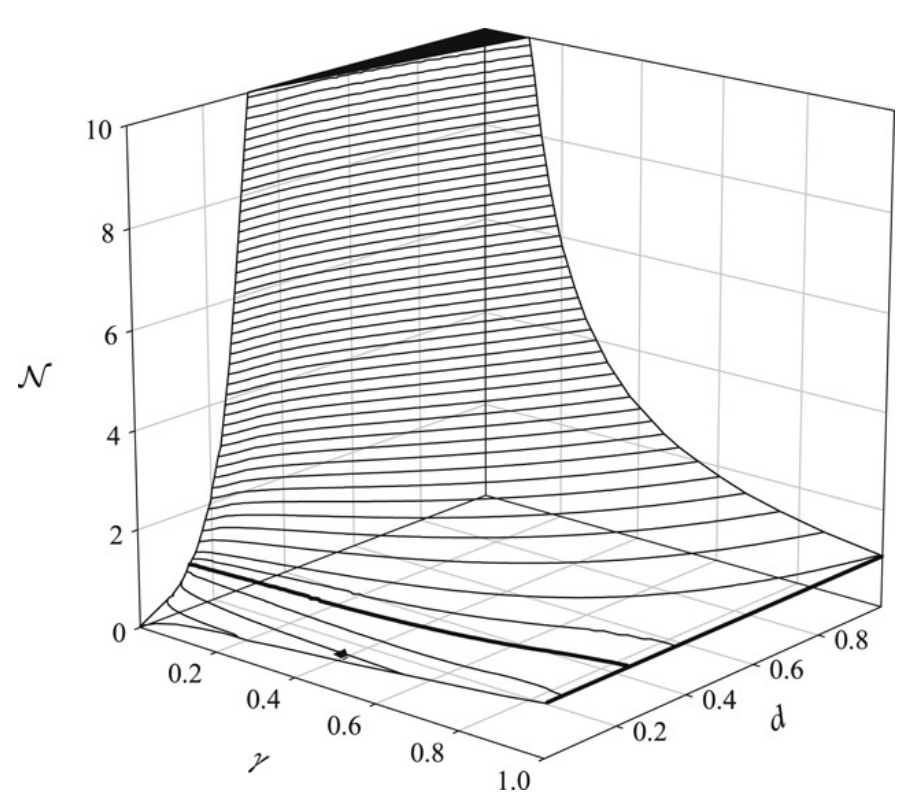

Fig. 7. The number $\mathcal{N}$ of monofractals within the phase-space volume $\gamma$ related to the specific dimension $d$ at the deformation parameter $\kappa=10$ (different levels $\mathcal{N}=$ const are shown with thin curves, thick lines relate to $\mathcal{N}=1$ ).

\section{Conclusions}

As the above consideration shows, the statistical mechanics of self-similar complex systems with phase space, whose specific fractal dimension $d$ is distributed with spectrum $f(d)$, is governed by the Tsallis formalism of the non-extensive thermostatistics. At that, the non-additivity parameter plays the role of an inverse multifractal function $\tau(q)=q d_{q}-f\left(d_{q}\right)$ which monotonically increases, taking value $\tau=0$ at $q=1$ and $\tau \simeq 1$ at $q \rightarrow \infty$ (the latter limit relates to the smooth phase space, where $\tau=1)$. The multifractal function $\tau(q)$ is reduced to the specific heat to determine, together with the inverse value $\bar{\tau}(q) \equiv 1 / \tau(q)>1$, both statistical distributions and thermodynamic functions of the system under consideration. At a given function $\tau(q)$, optimization of the normalized multifractal spectrum $f(d)$ arrives at the dependence of the statistical weight on the system complexity.

It is shown that the whole set of monofractals within a multifractal related to the phase space, which gives the support of a generalized thermostatistics, is modeled by the mass exponent $\tau(q)$ that determines the statistical weight (8) at a given volume $\gamma$. For the entropy (2) to be concave, Lesche stable et cetera, the exponent $\tau(q)$ should be a function, monotonically increasing within the interval $[0,1]$ at multifractal exponent variation within the domain $[1, \infty]$. The simplest case of such a function gives the hyperbolic tangent $\tau=\tanh (q-1)$ whose deformation (39) defined in accordance with both the Tsallis and Kaniadakis exponential functions (37) and (38) enable one to describe arbitrary multifractal phase space explicitly. At that, the Tsallis deformation arrives at more fast variations of the statistical weight exponent $\tau(q)$ in comparison with the non-deformed hyperbolic tangent, whereas the Kaniadakis one slows down these variations with increasing deformation parameter $\kappa$. All possible dependencies $\tau(q)$ are bounded from above by the linear function $\tau^{(T s)}=q-1$ at $q \in[1,2]$ which is transformed into the constant $\tau=1$ at $q>2$. This dependence relates to the smooth phase space within the Tsallis interval $q \in[1,2]$.

The dependence (6) of the number of monofractals within the phase-space volume $\gamma$ related to the multifractal with the specific dimension $d$ is determined by the spectrum function $f(d)$. This function increases monotonically, taking the minimum value $f=-1$ at $d=0$ and the maximum one $f=1$ at $d=1$; besides, its derivative equals $f^{\prime}(0)=\infty$ on the left boundary and $f^{\prime}(1)=1$ on the right one. The whole set of the spectrum functions is bounded by the limit dependencies $f^{(T s)}=2 d-1$ and $f^{(K)}=d$, the first of which relates to limit magnitude of the Tsallis deformation parameter $\kappa=1$ and the second one corresponds to the Kaniadakis limit $\kappa=\infty$. The number of monofractals within the multifractal increases with the $\gamma$ volume growth at small dimensions $d$ and falls down in the limits $d \rightarrow 1$ to give infinitely increasing at $\gamma \rightarrow 0$.

\section{Acknowledgement}

We thank anonymous referees for their constructive criticism. 


\section{References}

[1] M. Gell-mann, C. Tsallis, Nonextensive Entropy: Interdisciplinary Applications, Oxford University Press, Oxford, 2004.

[2] Naudts Jan, Physica A 340 (2004) 32.

[3] Garcia-Morales Vladimir, Pellicer Julio, Physica A 361 (2006) 161.

[4] L. Lyra, C. Tsallis, Phys. Rev. Lett. 80 (1998) 53.

[5] P. Jizba, T. Arimitsu, Ann. Phys. 312 (2004) 17.

[6] H. Touchette, C. Beck, J. Stat. Phys. 125 (2006) 459.

[7] T.C. Halsey, M.H. Jensen, L.P. Kadanoff, I. Procaccia, B.I. Shraiman, Phys. Rev. A 33 (1986) 1141.

[8] J. Feder, Fractals, Plenum Press, New York, 1988.

[9] C. Tsallis, R.S. Mendes, A.R. Plastino, Physica A 261 (1998) 534.

[10] S. Abe, S. Martínez, F. Pennini, A. Plastino, Phys. Lett. A 281 (2001) 126.

[11] S. Martínez, F. Nicolás, F. Pennini, A. Plastino, Physica A 286 (2000) 489.

[12] A. Olemskoi, S. Kokhan, Physica A 360 (2006) 37.

[13] C. Beck, F. Schlögl, Thermodynamics of chaotic systems, Cambridge University Press, Cambridge, 1993.

[14] G. Kaniadakis, Physica A 296 (2001) 405. 